\title{
A pilot study on yearlings' reactions to handling in relation to the training method
}

R. Polito, M. Minero *, E. Canali, M. Verga

Istituto di Zootecnica Veterinaria - Facoltà di Medicina Veterinaria - Università degli

Studi di Milano - Via Celoria, 10, 20133 Milano, Italy

Phone 0039 (0)2 50318042 - Fax 0039 (0)2 50318030

10

11 


\section{Abstract}

2 Handling and training methods of horses, which specially emphasize the importance of 3 understanding horse body language and the use of reinforcements ,are often used in practice, yet

4 their effects are not completely known. This study investigated whether the use of a sympathetic

5 approach during the preparation for public auctions influenced the reactivity of young horses

6 towards humans. Sixteen thoroughbred yearlings were prepared for the public auctions during

7 one month: eight horses ("Control") were handled according to conventional practices, while the

8 others ("Treated") were handled with two sessions of basic training based on body language. The

9 reactivity of horses was assessed in the presence of an "unfamiliar person" and a "familiar

10 person" inside the horse's box. The experimenter recorded the presence/absence of selected

11 behaviors during seven observational moments: "approaching the box", "opening the box door",

12 "entering the box" and four consecutive observations every thirty seconds. Reactivity of horses

13 was ranked during the first experience of "bit", "grooming", "shower" and application of the

14 "surcingle". Heart rate was telemetrically recorded during this final test. At the end of the auction 15 preparation, "Treated" horses exhibited more "contact" $(\mathrm{P}=0.08)$ and "lick" $(\mathrm{P}<0.05)$ behaviors in

16 the presence of a person. "Control" horses showed higher (NS) percentages of negative (more

17 nervous) rankings during "bit", "grooming" and "surcingle" tests. Two "Control" horses showed

18 aggressive behavior during the application of the surcingle and the test was interrupted to

19 guarantee person and animal safety. In this pilot study, horses handled with a sympathetic

20 approach showed less reactive behaviours compared to "Control" subjects. It would be interesting

21 to enlarge the sample size and assess if the use of a non coercive handling during the whole

22 training period influences their welfare positively and for a long time.

24 Keywords: Human-horse relationship; Behavior; Training; Handling; Heart Rate. 


\section{$1 \quad$ Introduction}

2 Many methods of horse handling are based upon traditional knowledge and do not actually

3 consider the specific ethogram of the horse. The interest in training methods that take into

4 account the natural behavior of the horse and avoid harsh methods has been increasing in the last

5 few decades in developed countries. These different methods, often defined as "sympathetic

6 horsemanship", emphasize the role of horse body language or the use of positive reinforcements

7 (Miller 1991; Roberts 1997; Waran, McGreevy and Casey 2002). Few scientific studies have

8 been published so far about the different training methods of horses. Shanahnan (2003) indicated

9 that non-aversive training, based on Tellington-Touch Equine Awareness Method (TTEAM)

10 reduces heart rate, saliva cortisol, loading time and stress during the loading of horses that are

11 difficult to transport. McGreevy (2004) reported that training is most effective when the related

12 practices consider specific learning abilities and the minimization of stress. An early handling is

13 more profitable, reduces emotionality and enhances learning ability (Heird et al. 1986; Mal and

14 McCall 1996), and reduces the prevalence of resistances and defensive aggressions (Miller 1991;

15 Spier et al. 2004). Jezierski et al. (1999) reported that foals handled for 10 minutes for 5 days a

16 week showed a lower heart rate and a better tractability during tests in comparison with non

17 handled foals. Heavy-handed or inexperienced riders can inadvertently cause pain to the horses

18 causing a conditioned fear response of avoidance (Casey 2002). Intensively handled foals are

19 calmer and more tractable than untreated ones (Simpson 2002), however their learning efficiency

20 can deteriorate if they are pushed to work too hard (Rubin, Oppergard and Hintz 1980).

21 Hence, it would be very important to assess objectively the effects of handling methods on horse

22 behavior. These studies would be innovative and useful because "sympathetic" training methods

23 that emphasize the importance of body language are increasing their popularity in horse practice.

24 However, their consequences on horse welfare are not known.

25 To reach this aim, the authors investigated whether the use of a sympathetic approach during the 26 preparation for public auctions influenced the reactivity of young horses towards humans. 


\section{Methods}

3 This study was carried out during a two month period at the S.A.B. (Società Allevamento

4 Besnate), one of the largest thoroughbred stud farms in Italy.

\section{2.1. Animals and Management}

6 Experimental subjects were 16 thoroughbred yearlings, (16-18 months old), balanced for sex,

7 taken to the stud farm for the auction preparation. The horses, prior to reaching the training

8 center, lived in groups in grass paddocks, interacting with humans only during feeding, monthly

9 weighing and veterinary treatments. After reaching the training center, they were individually

10 stabled in loose-boxes with a straw bed and a frontal sliding door. Horses were submitted to the

11 same daily management routine, water was available ad libitum and they were fed hay and

12 concentrate twice a day. The horses were randomly divided into two homogeneous groups,

13 subsequently named "Treated" and "Control" group.

\section{2.2. Experimental Procedures}

15 "Control" yearlings were traditionally handled by experienced stud personnel daily to become

16 habituated to humans. The procedure consisted in haltering, leading outdoors to the paddock,

17 brushing, picking up their feet and receiving veterinary examinations. Each "Treated" horse

18 received two sessions of "sympathetic training"(with an interval of a fortnight between the two)

19 following the procedure used by Roberts (1997) and briefly summarized hereafter. Every session

20 lasted from 15 to 45 minutes, in relation to horse reactions, and took place in an indoor $15 \mathrm{~m}$

21 circular walled pen. The experimenter (an experienced trainer) let the horse loose and encouraged

22 it to move round with the use of body posture and a length of lunge. When the yearling showed

23 signs of attention-like movements of the inside ear, the trainer turned his body at an angle of 45

24 degrees towards him and let it approach and follow, rewarding it with a gentle stroke. 
2 "Treated" and "Control" horses were observed during the following reactivity tests by personnel

3 who were not aware of which treatment each animal received:

4 1. in the presence of either an "unfamiliar person" or a "familiar person" in their usual box

5 ("reaction to a stationary human");

6 2. to the first experience of wearing a "bit", being groomed ("grooming"), being showered

7 ("bathing") and wearing a surcingle ("surcingle");

8 As the yearlings underwent the behavioral tests, they were video-recorded from a hidden place so

9 as not to interfere with their normal behaviour. The recorder's placement allowed observation of

10 the whole body of each horse regardless of its position and avoided any interference with the

11 tests.

\section{“Reaction to a stationary human”}

13 This test consisted of the direct observation of the horse's reactions to the presence of either an 14 "unfamiliar person" (UP) or a "familiar person" (FP) in his own box. UP and FP were two 15 women who wore blue overcoats and avoided direct eye contact during the tests. The 16 experimenters, using an instantaneous time sampling method, recorded the presence/absence of 17 selected behaviors at particular instants: "approaching the box", "opening the box door", 18 "entering the box" and during four consecutive observations every 30 seconds.

19 The following behaviors were recorded: immobile, approaching person, sniffing person, in 20 contact with person, licking person, nibbling person's clothes, strike threat, moving away from 21 person, ears back, bite threat, kick threat and rear threat.

22 Tests were made twice, with an interval of 32 days, and at least at two hours from feeding times.

23 The two replicates of the test are hereafter called Initial Test and Final Test. Two tests were 24 administered during each replicate: the first was performed by UP and the second, after 8 hours, 25 by FP. 
1 To assess the effects of handling on the reactivity of young horses towards specific management

2 practices, yearlings were observed during the tests described below.

$3>$ "Bit"

4 The handler introduced a bit without bridle into the mouth of each horse in its own box. The

5 horses were scored as "calm" when they accepted the bit without resistance or "reactive" if they

6 lifted their heads, recoiled or were reluctant to lower their heads.

$7>$ “ $>$ rooming"

8 The test was performed in the home boxes and consisted in 3 phases when the handler: 1. stroked

9 with a plastic curry comb and brushed the whole body of each horse; 2 . cleaned eyes, nose and

10 muzzle with a moist cloth; 3. brushed mane and forelock. During each phase, the horses were

11 scored as "calm" when they accepted the manipulations remaining immobile or "reactive" when

12 they tried to flee or showed an aggressive body posture (Waring 2003).

$13>$ “Bathing”

14 Each horse was led to the washing box. The test consisted in 4 phases: 1 . wetting of the whole

15 body; 2. shampooing with a sponge; 3. rinsing with the shower; 4. drying using a sweat scraper.

16 Horses were scored as "calm" or "reactive" as in the previous test.

$17>$ "Surcingle"

18 The "surcingle" test took place in an indoor round pen. Two people were necessary to perform

19 the test. One experienced handler restrained the horse with a head collar and a lead rope and the

20 other one fixed a surcingle and a heart rate monitor (Polar ${ }^{\circledR}$ Vantage NV). The handler: 1.

21 moistened the horse's coat at the electrodes' position on the cardiac area and the upper left

22 thorax; 2. placed the surcingle with the heart rate recorder.

23 Behavioral reactions of horses during both phases were classed as "calm" when they did not try

24 to escape and they had facial expressions of alert wakefulness (Waring 2003) for more than 50\%

25 of the time. They were considered "reactive" when they tried to flee, rear, bite or kick, they 
1 pawed, snorted and they had facial expressions of alarm (Waring 2003) for more than $50 \%$ of the

2 time.

3 Heart rate (HR, at $5 \mathrm{~s}$ intervals) and heart rate variability (HRV) were telemetrically recorded and

4 data were stored for future analysis. For each horse, recording periods without artifacts were

5 selected ( $8 \mathrm{~min}$ for $\mathrm{HR}$ and $5 \mathrm{~min}$ for HRV). HRV gives information about the sympathetic-

6 parasympathetic autonomic balance (Task Force of the ESC and the NASPE 1996). The

7 following time domain parameters were calculated (Marchant-Forde, Marlin and Marchant-Forde

8 2004): average inter-beat interval (IBI), maximum and minimum R-R waves intervals ( $R R \max$

9 and RRmin), standard deviation of the R-R intervals (SD) and the root mean square of successive

10 differences (RMSSD). Frequency domain analysis was performed and the following parameters

11 were calculated (Marchant-Forde, Marlin and Marchant-Forde 2004): LF (0.01 - $0.07 \mathrm{~Hz}$,

12 corresponding to the sympathetic nervous system activity), HF (0.07 $-0.5 \mathrm{~Hz}$, corresponding to

13 the parasympathetic autonomic nervous system activity) and LF/HF (corresponding to the 14 modulation of the sympathetic versus vagal branches).

15 Two "Control" horses exhibited aggressive behavior towards the experimenters hence their tests 16 were interrupted.

\section{2.4. Statistical Analysis}

18 Inter-observer reliability between experimenters was assessed by means of independent parallel

19 coding of a random sample of videotaped tests (10\%). Percentage agreement was always more

20 than $98 \%$. Behavioral data were statistically analyzed. Frequency and proportional duration of

21 each behavior were calculated. Behaviors that did not occur or that were recorded only

22 sporadically were not included in the statistical analysis. The behaviors that were included were:

23 "Immobile" “Approaching", "Sniffing”, "In Contact", "Licking Person" and "Nibbling”. Data

24 were then analyzed by means of the non-parametric analysis of variance test (Kruskal-Wallis

25 Test) (SPSS 2003). To analyze the interaction between behavioral variables of the test, a principal 
component analysis with Varimax rotation (PCA) was used. Factor scores were calculated for

2 horses when the component's Eigen value was greater than 1 (SPSS 2003).

3 For the "surcingle" test, integrated behavior and HR analysis were carried out. The HR curves

4 were visually analyzed and compared to the behavior of the horses, in order to verify if variations

5 in HR corresponded to specific reactions of horses or environmental stimuli. HRV was calculated

6 by means of the index method and the frequency method and the relevant data were analyzed by

$7 \quad$ Wilcoxon Match Paired test.

$9 \quad$ Results

“Reaction to a stationary human"

11 When comparing the data from the initial and final reactivity tests a change in the behavior of the

12 yearlings was noted after the handling period.

13 The results from the Principal Component Analysis (PCA) on the initial and final tests showed

14 that three components accounted for $25.1 \%, 20 \%$ and $16.5 \%$ of the variance.

15 The first component (PC1) is described by the variables "Immobile" (0.746), "Sniffing Person" 16 (0.628) and "Approaching" with negative sign (-0.860). These behaviors correspond either to an 17 exploratory attitude or to diffidence towards the experimenter. The second component (PC2) is 18 represented by behaviors that correspond to both high negative "Moving away" $(0.735)$ and high 19 positive "Nibbling Clothes" (-0.786) reactivity and may indicate some form of heightened 20 reactivity. The third component (PC3) is composed of "In Contact" (0.539) and "Licking 21 Person" (0.706) which are both behaviors indicating a positive attitude towards humans.

22 In this study the difference between the yearlings before and after handling was displayed mainly 23 on the second component (Fig. 2). Before handling, the yearlings mostly kept at a distance from 24 the experimenter while after handling they sought contact by biting the handler's clothes.

25 During the initial test there were no significant differences between "Control" and "Treated" 26 horses and during the final test "Treated" horses showed more licking behavior $(\mathrm{p}<0.05)$ in 
1 presence of the UP (Fig. 1a), and more contact $(p=0.09)$ and licking behavior $(p=0.1)$ with the FP

2 (Fig. 1b).

3 "Immobile" behavior was displayed mostly in the initial phase of the test and the percentage of

4 immobile yearlings decreased progressively from $42.9 \%$ ("approaching the box") to

$5 \quad 14,3 \%$ (“entering the box"), 3.6\% (1') and 0\% (after 1'30').

$6>$ “Bit”- “Grooming”-“Bathing” Tests

7 During the "Bit" test $87.5 \%$ of "Control" horses displayed highly reactive behavior as opposed to $837.5 \%$ of the "Treated" group. Highly reactive behavior was defined by the behavior the horses

9 displayed in an attempt to avoid the placement of the bit by backing up and lifting the head.

10 During all 3 phases of the "Grooming" test, "Control" horses showed more reactivity than

11 "Treated" horses.

12 During the use of the "Brush - Curry-comb" $25 \%$ of "Control" horses reacted by showing 13 avoidance behavior while none of "Treated" showed a reactive behavior. During the use of the 14 moist cloth the "Treated" group stayed calm whereas $37.5 \%$ of the "Control" group reacted 15 negatively. 50\% of the "Control" group and $12.5 \%$ of the "Treated" group displayed reactivity

16 while their mane-forelock was brushed.

17 "Control" horses showed more reactivity than the "Treated" horses during the 4 phases of 18 "Bathing" test, particularly during the first contact with water $(87.5 \%)$ and the "soap" phase $19(37.5 \%)$.

20 As for the "surcingle" test, during the "moist coat" phase $66.7 \%$ of "Control" and $25 \%$ of

21 "Treated" showed reactivity, while during the surcingle phase $22.2 \%$ of "Control" showed 22 reactivity as opposed to none in the "Treated" group.

23 Integrated analysis of HR and behavior established that noises that came from outside the round 24 pen were mostly the cause for the variation of HR, independently from the treatment condition.

25 For all the horses the highest peaks of HR were correlated to human voices, dog barks, neighing 26 of other horses and particularly to aeroplane noise (fig. 3) (the stud farm was near Malpensa 
1 airport). The state of alertness and HR variations were noted to happen contemporaneously. Table

21 features the HRV average values and reports the comparisons between the "Treated" and

3 "Control" groups. No significant difference was found in any of the parameters.

\section{Discussion}

6 The aim of this pilot study was to investigate through behavioral tests the effects of sympathetic

7 handling on the reactivity of young horses towards humans and on their first experiences of 8 specific manipulations.

9 The analysis of the horses' reactions to a stationary human showed that, particularly after the

10 handling period, both groups exhibited behaviors related to the exploration and investigation of

11 the person present in the box during the test by displaying the following behaviors: "Sniffing",

12 "In Contact" and "Nibbling". Fraser (1992) reports that horses show exploratory behavior

13 exclusively when they are not experiencing fear and apprehension. Therefore, it seems that the

14 horses that participated in this experiment were not fearful of humans during this phase of the 15 trial.

16 In "Treated" yearlings, "Licking Person" and "In Contact" occurred more often during the tests

17 showing a higher tendency to seek contact with humans. "Nibbling Clothes" was among the most

18 frequently exhibited behaviors and was not considered as a bite but as a sign of curiosity and

19 exploration. Nibbling an object is one of the first play responses associated with approaching and

20 investigating an object (McDonnel and Poulin 2002). The occurrence of this behavior can be

21 explained by the combination of the curiosity that is typical of young horses together with the

22 acceptance of humans during the training sessions. Licking and smelling an inanimate object is

23 used to investigate the smell, the structure, the shape, the taste and the dimensions of the object

24 but also precede and/or are performed at the beginning of reciprocal grooming between the 25 individuals of a herd (McDonell and Poulin 2002). 
1 Among the explorative behaviors, "Approaching" and "Seeking Contact" are often induced by

2 the sight of familiar objects in an unfamiliar environment or vice versa. By exploring, the horse

3 acquires useful and important information which results in high adaptability causing the horse to

4 respond promptly when necessary (Fraser 1992).

5 In the present study, explorative behavior was displayed by both groups and more frequently in

6 "Treated" horses. "Immobile" behavior was recorded more often in the initial phases of the test.

7 This behavior may be interpreted as a pause before approaching the experimenter: the horse waits

8 immobile verifying that there is no danger and starts moving only when everything is under

9 control. In this case "Immobile" behavior could be due to the horse being accepting and relaxed

10 in the presence of a human and not a behavior that would be displayed in a situation of extreme

11 fear or acute stress (freezing) (Archer 1973).

12 In both groups ,the PCA showed an increase in the occurrence of the behaviors associated with

13 the interactions with humans: "Approaching", "In Contact", "Sniffing Person" and "Nibbling

14 Clothes". Between the Initial and Final Tests, the horses became more curious and "relaxed"

15 when in presence of the experimenter. Grandin (1993) reported that animals used to frequent

16 manipulations and close contact with humans were more calm and less stressed than those who

17 rarely saw humans. It can be hypothesized that these behaviors are associated not only to the

18 handling but also to normal physical and behavioral development of foals that grow with

19 exposure to human contact (Waring 2003).

20 "Bit", "Grooming" and "Bathing" tests confirmed that "Control" horses showed higher

21 percentages of reactive behaviours. These manipulations are commonly used to prepare horses

22 for auctions and involve contact with some of the horses' vulnerable zones, like the head and the

23 abdomen.

24 The manipulations (similar to allogrooming) and the physical contact during the sessions of 25 sympathetic training may have facilitated the application of the harness and the management 
1 practices. "Control" horses reacted more negatively towards humans also during the first

2 application of the girth belt which induced the experimenters to interrupt two tests.

3 The occurrence of noises from the outside was correlated with an HR increase, alertness, high

4 attentiveness and stillness which are behaviors that animals show in conditions of fear or acute

5 stress (Archer 1973). HR rose and reached a peak when the intensity of the noise was highest.

6 These observations are in agreement with the results reported by Stewart et al. (2003) from their

7 study on air transport of horses, whereby one of the possible causes of HR increase was the

8 vibrations made by aeroplanes.

9 The occurrence of the variations in HR and behavior in this case shows that the animals were not

10 habituated to this type of acoustic stimuli. It therefore seems important to pay particular attention

11 to this type of environmental stimuli when rearing yearlings.

12 There were no statistically significant results from the analysis of the data on HRV. This might

13 have been due to high individual variability, to the limited number of horses being examined and 14 requires further investigation with a broader sample.

\section{Conclusions}

17 This pilot study revealed that after having been handled for a period of time the yearlings were

18 more sociable towards humans. The "Treated" group horses in particular showed more positive

19 interactions with humans resulting in lower reactivity and higher compliance during specific

20 manipulations in the preparations for the auction sales.

21 It would be of interest to investigate in further studies with larger animal samples if a sympathetic

22 training has a positive influence on the behavior and reactivity of the horses when used

23 throughout the training phase. Investigating if these horses perform better during races than

24 horses trained with traditional methods would be of equal interest. 


\section{Acknowledgements}

\section{References} Science 62: 1-11. 78: 263-290. Inc.

We thank P. Crespi and the personnel of the stud "SAB" for allowing use of the animals and facilities and V. Clerici for her help and work with horses.

Archer, J. 1973. Tests for emotionality in rats and mice: a review. Animal Behaviour 21: 205-235.

Casey, R. A. 2002. Clinical problems associated with the intensive management of performance horses. In The welfare of horses, ed. N. Waran. Dordrecht: Klewer Academic Publishers. Fraser, A. F. 1992. The behaviour of the horse. Wallingford: CAB International.

Grandin, T. 1993. Teaching Principles of Behavior and Equipment Design for Handling Livestock. Journal of Animal Science 71: 1065-1070.

Heird, J.C., Whitaker, D.D., Bell, R.W., Ramsey, C.B. and Lokey, C.E. 1986. The effects of handling at different ages on the subsequent learning ability of 2-year-old horses. Applied Animal Behaviour Science 15: 15-25.

Jezierski, T., Jaworski, Z. and Gorecka, A. 1999. Effects of handling on behaviour and heart rate in Konik horses: comparison of stable and forest reared youngstock. Applied Animal Behaviour

Mal, M.E. and McCall, C.A. 1996. The influence of handling during different ages on a halter training test in foals. Applied Animal Behaviour Science 50: 115-120.

Marchant-Forde, R.M., Marlin, D.J. and Marchant-Forde, J.N. 2004. Validation of a cardiac monitor for measuring heart rate variability in adult female pigs: accuracy, artefacts and editing. Physiology and Behavioural 80: 449-458.

McDonnell, S.M. and Poulin, A. 2002. Equid play ethogram. Applied Animal Behaviour Science

McGreevy, P. 2004. Equine Behavior. Journal of Equine Veterinary Science 24: 397-398.

Miller, R.M. 1991. Imprint training of the newborn foal. Colorado Springs: Western Horseman

Roberts, M. 1997. The man who listens to horses. London: Arrow Books.

9 Rubin, L., Oppergard, C. and Hintz H.F. 1980. The effect of varyng the temporal distribution of conditioning trials on equine learning training. Journal of Animal Science 50: 1184-1187.

Shanahan, S. 2003. Trailer loading stress in horses: behavioral and physiological effects of nonaversive training /TTEAM). Journal of Applied Animal Welfare Science 6: 263-2744.

33 Simpson, B.S. 2002. Neonatal foal handling. Applied Animal Behaviour Science 78: 303-317. 
1 Spier, S.J., Pusterla, J.B., Villarroel, A. and Pusterla, N. 2004. Outcome of tactile conditioning of

2 neonates, or "imprint training" on selected handling measures in foals. The Veterinary Journal

3 168: 252-258.

4 SPSS 2003. Command Syntax Reference. Chicago: SPSS Inc.

5 Stewart, M., Foster, T.M. and Waas, J.R. 2003. The effects of air transport on the behaviour and

6 heart rate of horses. Applied Animal Behaviour Science 80: 143-160.

7 Task Force of the European Society of Cardiology and the North American Society of Pacing and

8 Electrophysiology 1996. Heart Rate Variability: Standards of Measurement, Physiological

9 Interpretation and Clinical Use. Circulation 93: 1043-1065.

10 Waran, N., McGreevy, P. and Casey, R.A. 2002. Training methods and horse welfare. In The

11 welfare of horses, 151-180, ed. N. Waran. Dordrecht: Klewer Academic Publishers.

12 Waring, G.H. 2003. Horse behavior. Park Ridge: Noyes Publications. 
Reactivity Test

4

5

6

7

8

9

10

11

12

13

14

15

16

17
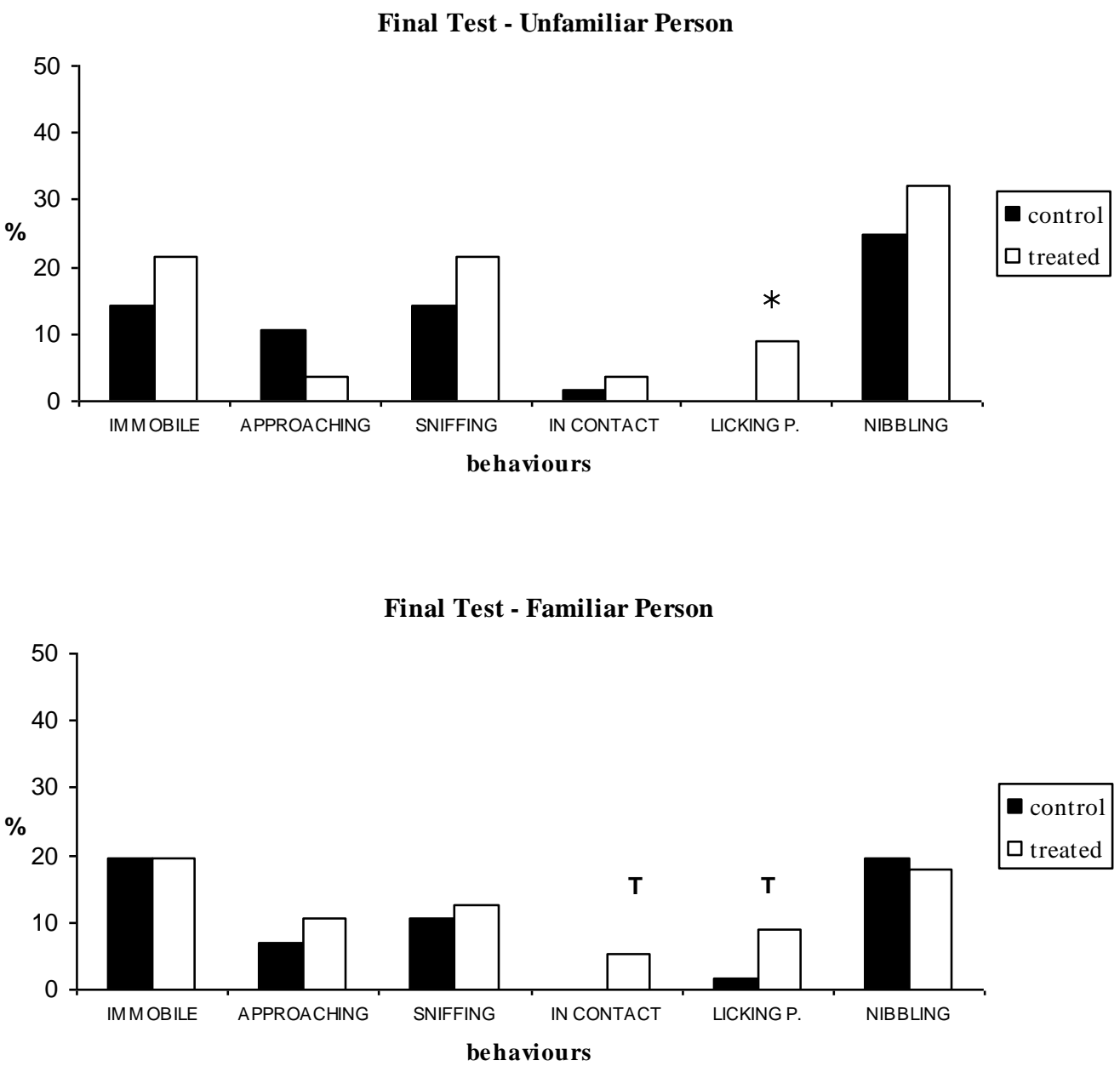

Fig 1a and 1b: Percentage of horses showing the observed behaviors. a) Unfamiliar Person. b) Familiar Person. $*(\mathbf{p}<0.05), \mathbf{T}(\mathbf{p}<0.1)$. 


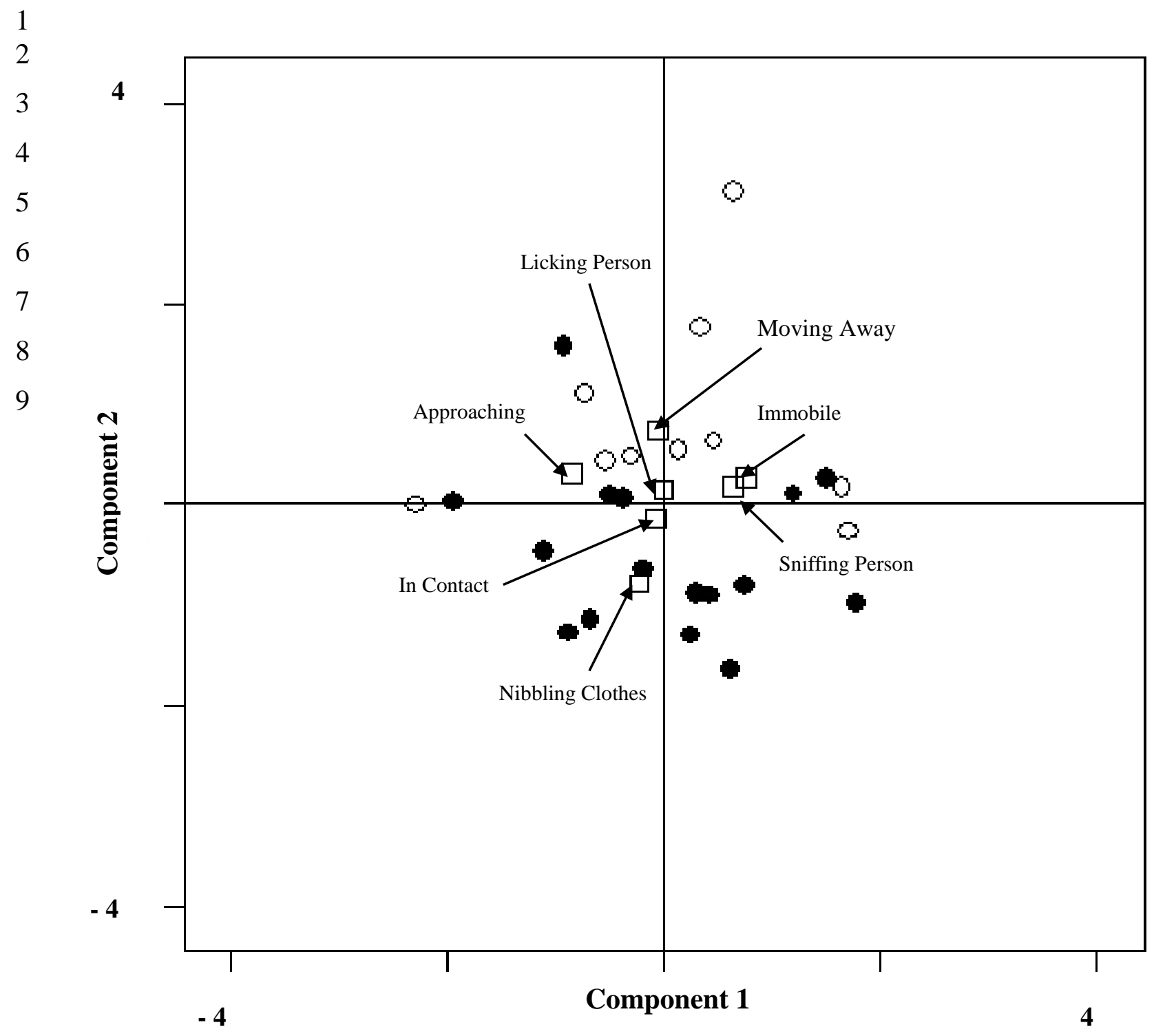

Fig 2: Projection for the loadings of the behavioral variables and yearlings' scores considered on the First and Second Principal Component. ( $\circ$ yearlings before treatment - yearlings after treatment - $\square$ behavioural variables)

TABLE 1: Principal Component Analysis (PCA) of behaviour

\begin{tabular}{llll}
\hline Behaviour & \multicolumn{1}{c}{ PC1 } & \multicolumn{1}{c}{ PC2 } & \multicolumn{1}{c}{ PC3 } \\
\hline Standing immobile & $\mathbf{0 . 7 4 6}$ & 0.259 & -0.272 \\
Approaching person & $\mathbf{- 0 . 8 6 0}$ & 0.318 & 0.109 \\
Sniffing person & $\mathbf{0 . 6 2 8}$ & 0.191 & 0.134 \\
In contact with person & -0.084 & -0.128 & $\mathbf{0 . 5 3 9}$ \\
Licking Person & -0.010 & 0.143 & $\mathbf{0 . 7 0 6}$ \\
Nibbling person's clothes & -0.241 & $\mathbf{- 0 . 7 8 6}$ & -0.324 \\
Moving away from person & -0.063 & $\mathbf{0 . 7 3 5}$ & -0.395 \\
\hline
\end{tabular}

${ }^{1}$ The most significant behaviours for each component are bold typed 


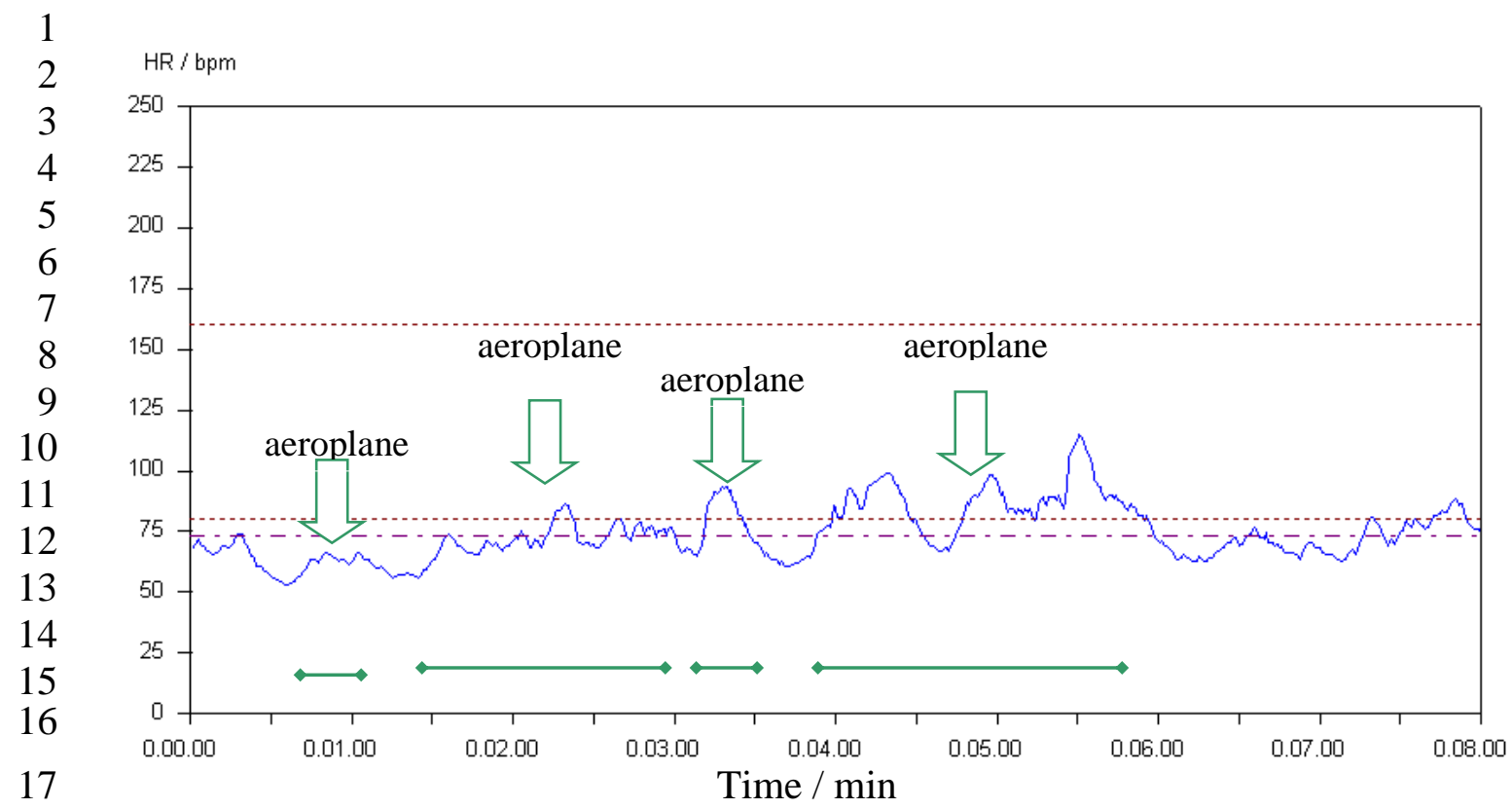

18 Fig 3: Mean HR over time of a yearling during the surcingle test

19

TABLE 2: HRV: time domain and frequency domain parameters during the surcingle test 23

\begin{tabular}{lccccccc}
\hline & \multicolumn{4}{c}{ Time Domain Parameters } & \multicolumn{3}{c}{ Frequency Domain Parameters } \\
\hline & R-R max & IBI & R-R min & RMSSD & LF & HF & LF/HF \\
\cline { 2 - 8 } Treated & 1359.3 & 1089.3 & 788.3 & 54.7 & 1818.2 & 725.1 & 43.4 \\
Control & 1359 & 1134.5 & 834.7 & 33.7 & 1259.0 & 421.6 & 42.1 \\
\hline
\end{tabular}

24

25

26 